\title{
MPPT using novel FLC based MPO for photovoltaic system
}

\author{
R. Arulmurugan \\ Department of Electrical and Electronics Engineering S R Engineering College, Warangal, India
}

\begin{tabular}{l} 
Article Info \\
\hline Article history: \\
Received Nov 11, 2018 \\
Revised Jan 11, 2019 \\
Accepted Jan 25, 2019 \\
\hline Keywords: \\
Enhanced perturb and observe \\
method \\
Fuzzy logic controller \\
Maximum power point tracking \\
Photovoltaic
\end{tabular}

\begin{abstract}
In this paper introduces a novel Fuzzy Logic Controller (FLC) as a Maximum Power Extracting (MPE) of photovoltaic standalone system is proposed, which can handle the uncertainties of the rules under high deviations in climate conditions. Conventional Perturb and Observe (P \& O), hill-climbing MPE structures are examined. The MPE employed boost converter controller. The new controller improves $\mathrm{P} \& \mathrm{O}$ based maximum power tracker search method by rules fuzzifying of such method and eliminates the conventional drawback. A new FLC based improved P \& O offers an accurate and fast converging to MPE during steady state and changing climatic situation compared to existing $\mathrm{P} \& \mathrm{O}$ and hill climbing method. The performance of the designed MPE is demonstrated in simulation MATLAB at dissimilar operating circumstances.
\end{abstract}

Copyright $(2019$ Institute of Advanced Engineering and Science. All rights reserved.

Corresponding Author:

R. Arulmurugan,

Department of Electrical and Electronics Engineering,

S R Engineering College,

Anandhasagar (V), Hansparthy (H), Warangal- 506 371, India.

Email: arul.lect@gmail.com

\section{INTRODUCTION}

The dc to dc converter uses to changes the output voltage according to its duty cycle. In general the buck-boost and buck converter lose half of their input power due to input current series switching. For that the boost converters should be excluded from extreme tracking applications. The boost dc to dc converter has continuous input current, but the output voltage is always higher than the input which may not attain maximum power transfer function in few cases, maximum power voltage is less than input. The MPPT approach traces the possible higher power via decreasing or increasing current and voltage [1]. Hence, the use of boost converter is effective as it decreases or increases current at voltage's expense and it's also able to exploit all the surviving power from photovoltaic due to its continuous input current $[2,3]$.

Maximum power extracting algorithm represents best load for PV panel, generating appropriate voltage for the load. The photovoltaic array yields exponential curves for voltage and current, where the supreme power arises at the curve's mutual knee [4]. The photovoltaic power and voltage characteristics are nonlinear and affected by the temperature and irradiance differences [5]. The applied maximum power tracking uses a type of controller and reasoning to look for the knee, which, in turn, allows the dc to dc boost converter to extract the extreme power from the photovoltaic panel $[6,7]$. The tracing technique offers a new reference signal for the control and excerpts the extreme power from the photovoltaic panel. Literature has recommended numerous MPPT methods [8,9]. The Incremental Conductive (INC) technique is based on the derivative of power over voltage or current being zero at the maximum power point, negative on the right of the MPP and positive on the left of the peak point [10]. This technique requires complex calculation to give good performance under quick weather situations change. Besides, the following peak power time is comparatively long for minor step size [11]. Hill-climbing technique workings by perturbation of the photovoltaic scheme which variations the power converter duty cycle and detects it on the output power, and then determining the new way of the duty-cycle to excerpt extreme power. The hill-climbing technique has 
slow reaction particularly under changing weather circumstances because the maximum power point tracking gives the choice directly for the duty cycle by announcing a controller of error signal. The current/voltagebased MPE approximates the ratio between the maximum power current/voltage and the short circuit current/open circuit voltage under dissimilar weather circumstances [12]. Perturb and observe technique is the usually used due to its simplicity, low cost and ease of implementation [13]. Perturb and observe works efficiently under changing weather circumstances where it can reach the error signal due to its parting between the MPE technique that controls the reference signal and the duty-cycle ensuing from varying the reference signal. Hence, perturb and observe employs the MPE for the reference signal, while the dc to dc converter can be controlled separately.

Among dissimilar artificial intelligent controllers, FLC is the simplest to integrate with the structure. Lately, FLC has received an increasing attention from researchers for motor drives, converter control, and other process control as it offers better reactions than other traditional controllers [14-20]. The inaccuracy of the weather changes that can be reflected by photovoltaic arrays can be exposed accurately using fuzzy logic based controller. In order to take the merits of FLC algorithm, the maximum power point tracking algorithm is integrated using fuzzy based controller so that the complete control system can always deliver maximum power transfer from photovoltaic panel to the converter side, in spite of the unpredictable climate circumstances. The problem of most of the FLC-based MPE algorithms [15-18] is that the tracing point is situated away from the MPE when the climate conditions variation. Moreover, the MPE tracking control depending on duty-cycle variations causes negligence in power converter error signal controller. Though, there is an essential to control the duty cycle of the charge converter and to trace the MPE depending on reference signal, not duty cycle [16-20]. This paper presents a new fuzzy logic control-based improved $\mathrm{P} \& \mathrm{O}$ technique for MPE standalone photovoltaic system. The designed MPE tracking is capable of exploiting the merits of the perturb and observe method and eradicates its problems. The MPE is analyzed by converting the improved $\mathrm{P} \& \mathrm{O}$ algorithm into 37 fuzzy rules after the controller two inputs and single output have been divided to seven fuzzy subsets. As the designed technique always transfers extreme power from photovoltaic arrays, it enhances the number of photovoltaic modules. The designed system is instigated in real-time using microcontroller board.

\section{IMPROVED PERTURBATION AND OBSERVATION METHOD}

The conventional Perturbation and Observation (P\&O) algorithm is working on the subsequent principle: if the voltage or current of the photovoltaic array is perturbed in a particular direction and the photovoltaic power rises, this means that the functioning point has traced toward the peak power and, hence, the functioning voltage or current must be perturbed in the similar direction. Else, if the power reductions, the functioning point has progressed away from the peak power and, so, the route of the functioning voltage or current perturbation must be inverted [17, 21].

Perturb and observe functions by varying the power converter voltage reference signal or duty-cycle and detecting its impact on the output power [18]. The perturbation and observation is the most usually used system because of its ease of implementation, simplicity and low cost. But, it fluctuates around the functioning point which causes losses in the power, and the functioning point traces away from the peak power point which loses the way during fast weather (radiance) changes. Due to the problems the swing around to this peak point [19].

Figure 1 illustrations the behavior of the photovoltaic power using the traditional $\mathrm{P} \& \mathrm{O}$ MPE technique. The photovoltaic output power is imposed to track toward the MPE point. After attainment the best point, the photovoltaic output power fluctuates around the point. At the period 0.33 seconds, the radiation reduced; so, power deviates from the best value due to the problem aforementioned.

The conventional $\mathrm{P} \& \mathrm{O}$ algorithm may exhibit erratic behavior in fast varying atmospheric circumstances as a result of moving clouds. This limitation can be illuminated using Figure 2 with a two voltage versus power curves with changing radiance [20]. Assume that the functioning point is at the point ' $A$ ' and is fluctuating around the maximum power point and a perturbation will moves the functioning point toward the point 'B'. Nevertheless, if the radiance rises rapidly to curve P2 power curve within single sampling period, the functioning point will really moves from the point ' $A$ ' toward the point ' $C$ '. This difficult occurs because the maximum power point tracking cannot recognize that power increment is the result of the rising radiation and simply assumes that it is the result of moving the functioning point to the maximum power point. If the radiance is still rapidly rising, the MPE continuing to perturb to the right again and the functioning point continues to deviate from the real MPE until the solar irradiation alteration slows. This condition can occur on the partly cloudy days, and the maximum power point extracting is most problematic because of the frequent movement of the maximum power extracting point. 
The Divergence of the conventional P \& O from the maximum power point under rapidly varying atmospheric circumstances that was defined in the previous section can be solved by utilizing the photovoltaic voltage-current curve. As shown in this curve, in fixed radiation, when the voltage decreases (increases), the current is increases (decreases). Using this simple statistic, can be resolved the Divergence from the real MPE in rapidly varying atmospheric situations, as follow. If the increases voltage and the increases instantaneously increase and the current increases too, the algorithm realizes that it is in rapidly varying atmospheric situations and decreases the voltage, instead, it increases. When the voltage and the power are increasing simultaneously and the current is decreasing, algorithm is in fixed irradiation and increases the voltage. Hence the MPE tracking algorithm avoids different from the actual MPE. The improved $\mathrm{P} \& \mathrm{O}$ algorithm flowchart is offered in Figure 3.

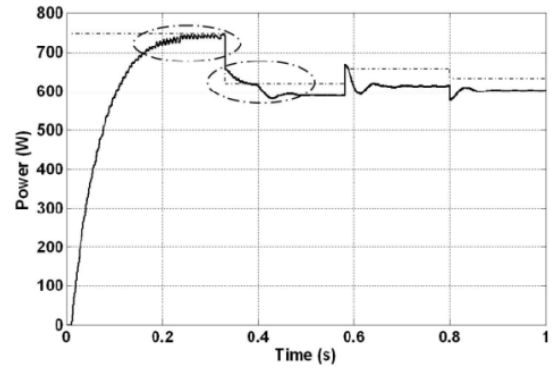

Figure 1. Photovoltaic output power of the conventional $\mathrm{P} \& \mathrm{O}$ approach

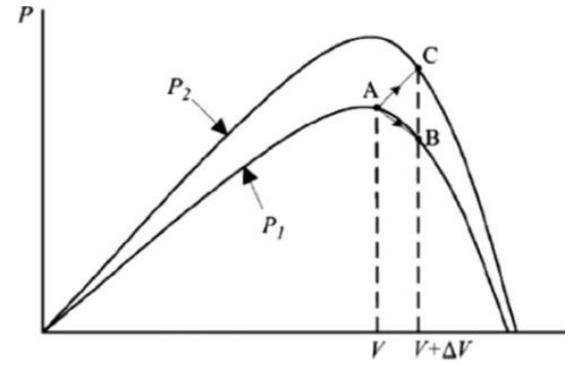

Figure 2. Variance of conventional P\&O from MPE as shown in

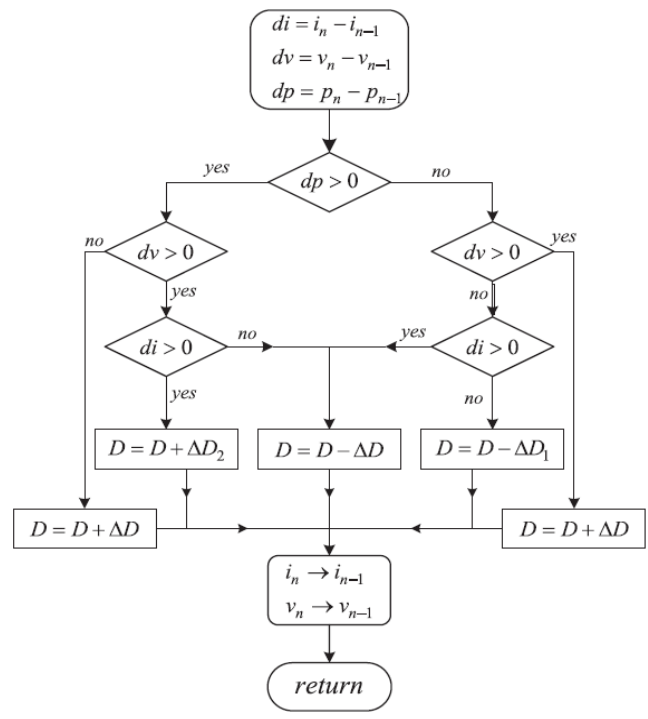

Figure 3. Improved P \& O algorithm flowchart as shown in [2]

\section{SUGGESTED NOVEL FUZZY SYSTEM}

The change of voltage output fed to the battery and convert into AC by using inverter is the primary operation of the DC to DC boost converter. In this process duty-cycle level decreases or increases depending on the maximum power. In addition, the controller variations the voltage level of output by changing the duty-cycle of the PWM signal, which traces the reference signal. A triangular reference signal is likened with the output signal of fuzzy voltage reference to produce a supposedly zero error signal. The fuzzy rules are derived from improved perturb and observe method. This reference signal is adaptive, varying its shape according to climatic situations. The boost converter feed the battery load with the most suitable power.

The proposed entire block diagram Figure 4 is shows of boost dc to dc converter together with the maximum power point tracking and the fuzzy logic controller. The creation of FLC was done using Mamdani technique. The design of the rules, membership functions will be detailed in the next sections. The Pulse Width Modulation PWM) changes its duty-cycle according to the control signal. 


\section{IMPROVED P\&O BASED NEW FUZZY LOGIC BASED METHOD}

In fuzzy logic controller design, one should detect the foremost control variables and regulate the sets that define the values of individually linguistic variable. The improved Perturb and Observe $(\mathrm{P} \& \mathrm{O})$ searching algorithm is calculated to attain the merits of conventional P \& O simplicity and eradicate all aforementioned disadvantages. The change in photovoltaic output voltage and the change in photovoltaic array output power are the two input of the modified FLC approach. The increment of the reference output voltage of the FLC where fed into discrete PI controller through Pulse Width Modulation (PWM) control box to produce the pulse. The two inputs and single output of the fuzzy logic controller are given in the equations from (1) to (3).

$$
\begin{aligned}
& \text { Del_P }=P(k)-P(k-1) \\
& \text { Del_V }=\mathrm{V}(\mathrm{k})-\mathrm{V}(\mathrm{k} 1) \\
& \text { Del_Vref }=\operatorname{Vref}(\mathrm{k})-\operatorname{Vref}(\mathrm{k} 1)
\end{aligned}
$$

The merits of this improved in perturb and observe is that the output of the fuzzy based control changes the reference voltage only. Hence, the duty cycle of the DC to DC boost converter can further be regulated using specific controller. Moreover, the boost converter controller ensures that the photovoltaic output power does not deviate from the maximum power point during changing weather situations or variable load.

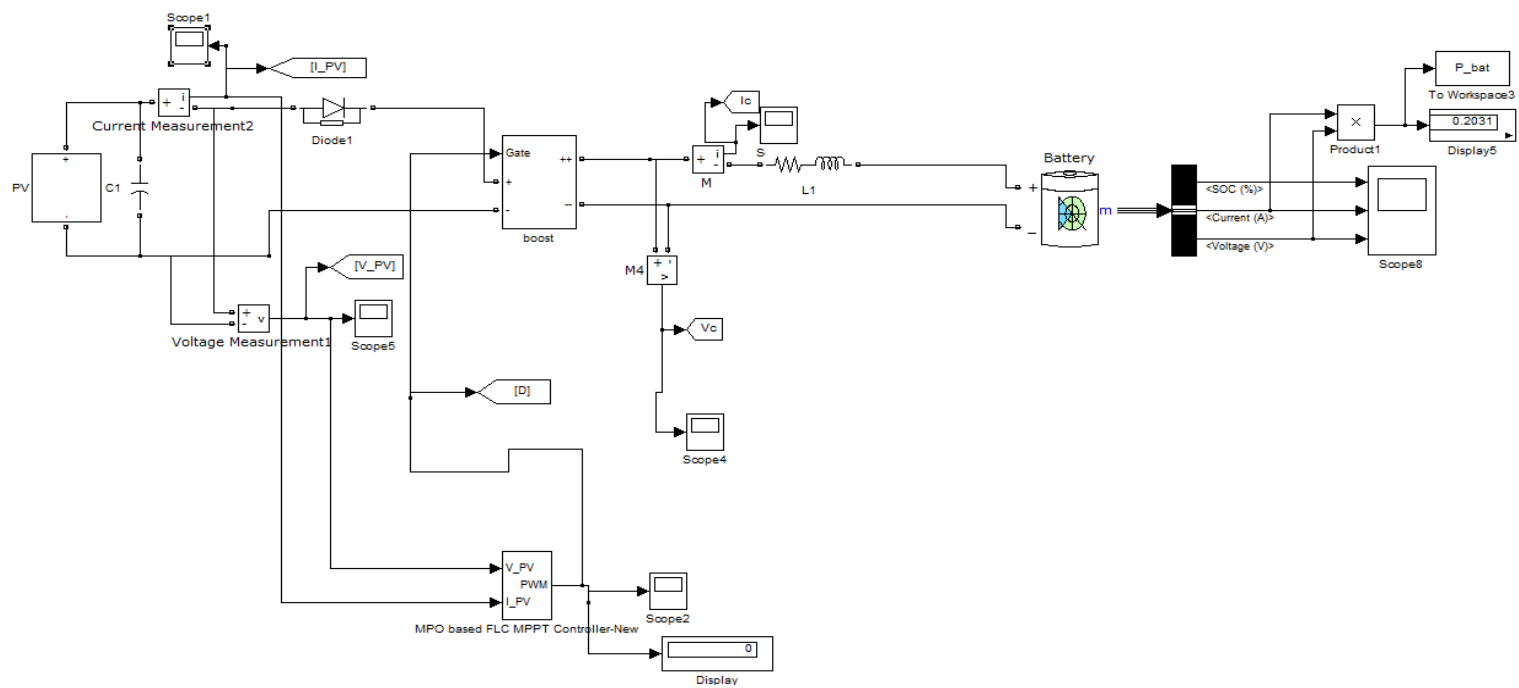

(a)

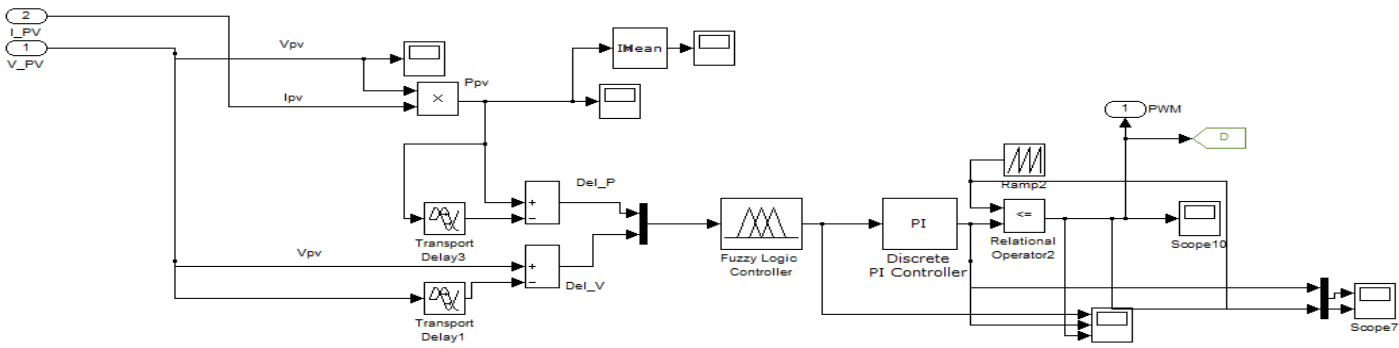

(b)

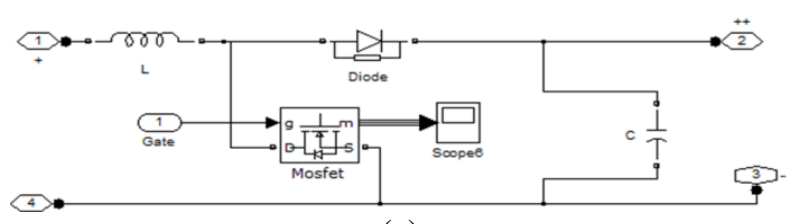

(c)

Figure 4. (a) proposed overall setup of the PV system (b) Subsystem of the Fuzzy logic controller (c) DC to DC Boost converter 
The variables of the input fuzzy logic are divided into seven fuzzy subsets which are: negative big (NB), negative medium (NM), negative small (NS), zero (Z), positive small (PS), positive medium (PM), positive big (PB). These seven fuzzy subsets for two input variables can produce forty-nine FLC rules, but the zero membership rules are shortened in one rule which is only making total of thirty-seven rules, instead of forty-nine. The Membership Functions (MF) of the output variables are nine-term fuzzy sets with classical triangular and trapezoidal shapes, positive double big(PBB), negative double big(NBB), negative big (NB), negative medium (NM), negative small (NS), zero (Z), positive small (PS), positive medium (PM), negative very small (NSL), positive very small (PSL) and positive big (PB). The fuzzy technique used here is Mamdani approach, where the max-min composition method is used for the inference and the center-ofgravity approach is used for the defuzzification procedure to convert the fuzzy subset reference voltage that changes to real numbers as offered in (4).

$$
\Delta V_{\text {ref }}=\frac{\sum_{i}^{n} \Delta V_{\text {ref } i} \mu\left(\Delta V_{\text {ref } i}\right)}{\sum_{i}^{n} \mu\left(\Delta V_{\text {ref } i}\right)}
$$

Where Vref is the FLC output and Vrefi is the output MF (Membership Function) center of maximumminimum inference composition.

The fuzzy logic control rules mimic the behavior of improved perturb and observe technique. The fuzzification of the P\&O technique with the rules is revealed in Figure 5. The shapes and fuzzy subset partitions of the MF in both output and two inputs exposed in Figure 6 depend on the behavior of the controller input and output signals. The fuzzy logic control deals with variable step size to decrease or increase the reference voltage; hence, the tracking period becomes little and the system performance during steady-state situations is much better than with traditional perturb and observe method. Furthermore, the zero -MF keeps the scheme in the steady-state without fluctuations once it attains the MPP; this zero -MF is considered an overtaking on perturb and observe approach in solving the problem of fluctuation. After Matlab simulaton of the improved perturb and observe algorithm with boost dc to dc converter the error (del_P) and change of error (del_V) is calculated and normalized between (-10 to 10), $(-0.5$ to 0.5$)$ respectively. The change in voltage reference (del_Vref) is normalized (-10 to 10). The fuzzy membership function used in this design is mamdhani method. In the new fuzzy based MPPT Gaussian surface fuzzy membership function is utilized. The triangular fuzzy MF for two input and single output are illustrated in the rule viewer and the corresponding surface diagram is shown in Figure 7 and Figure 8 respectively.
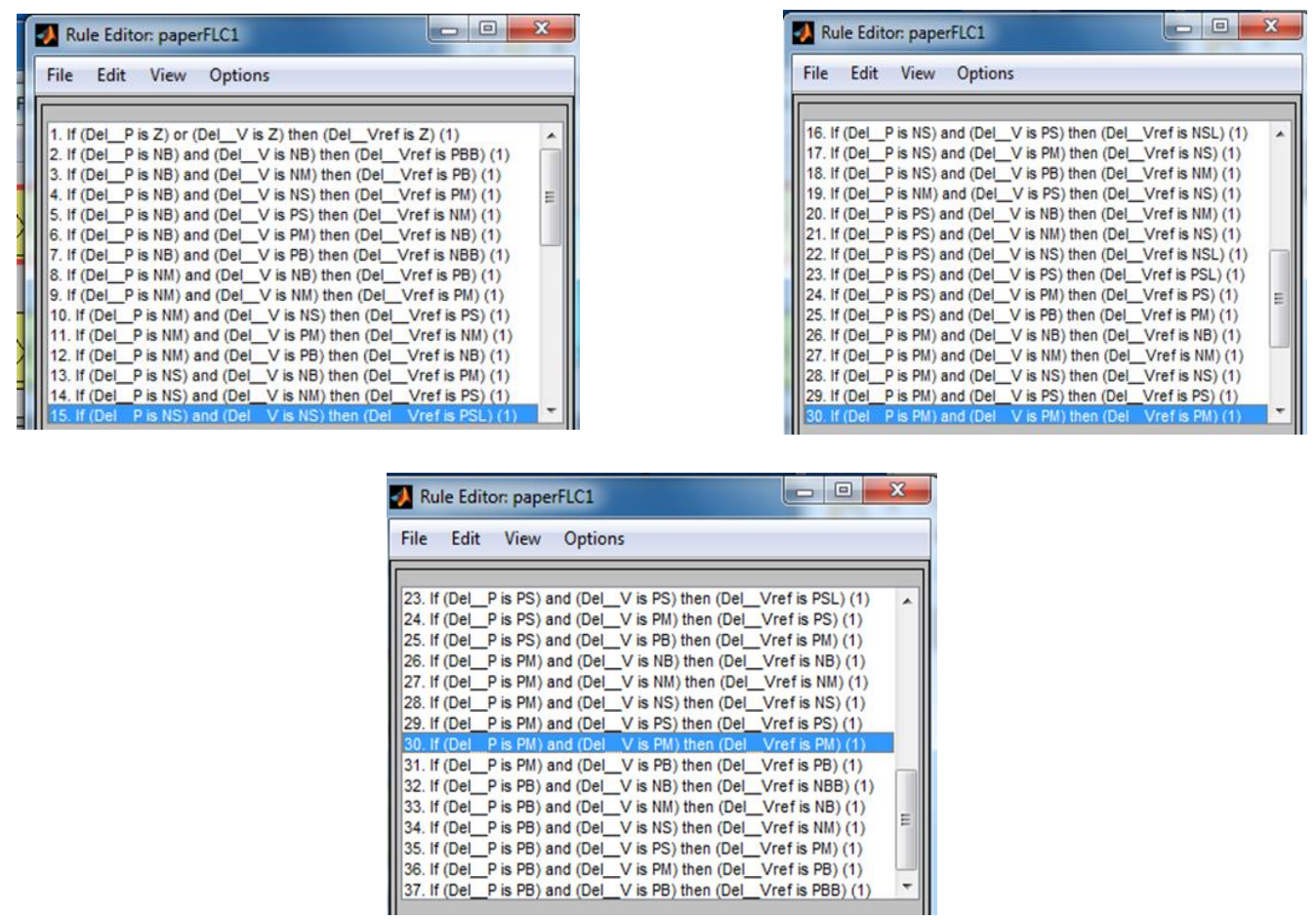

Figure 5. Fuzzification of the improved perturb and observe rules 

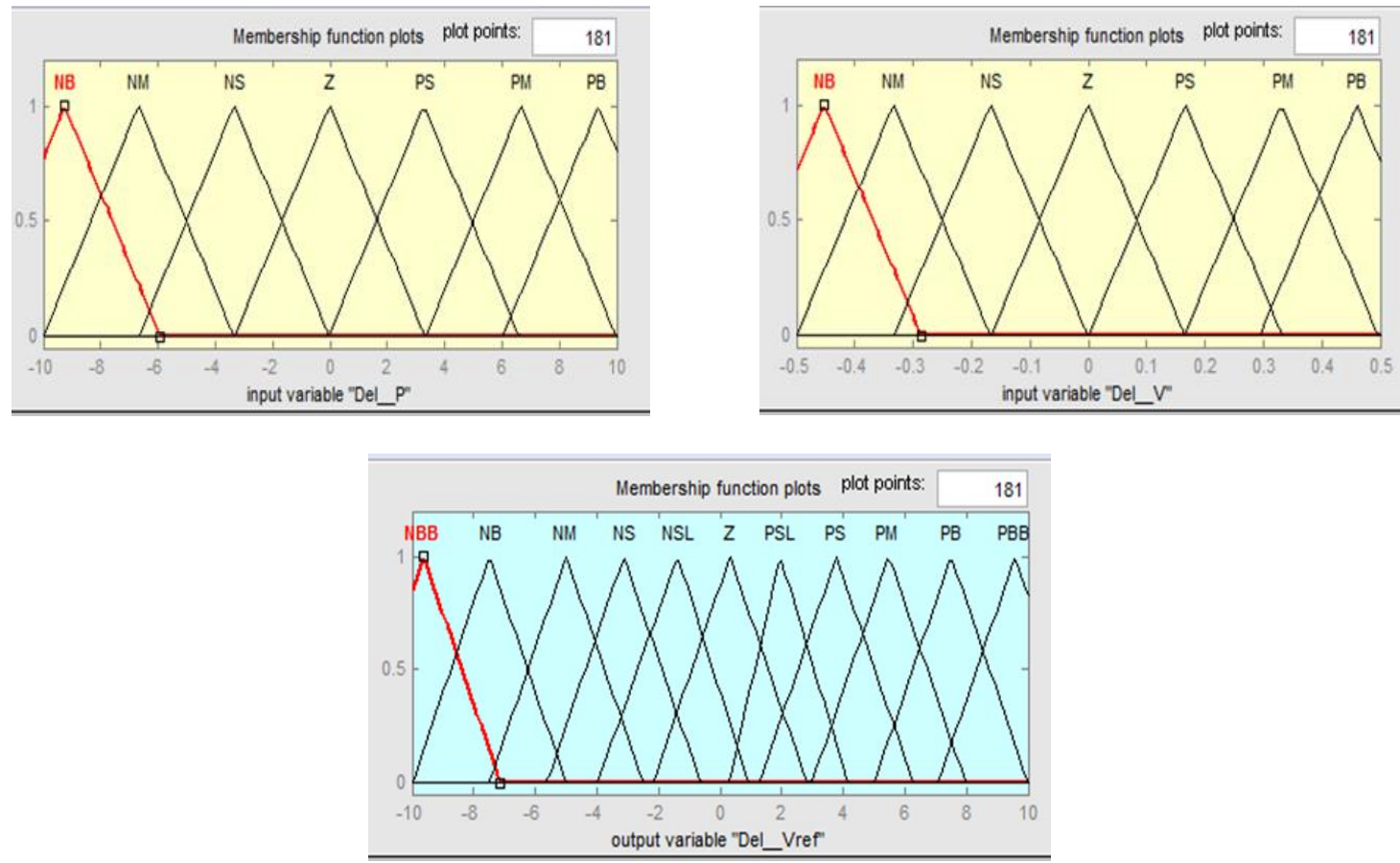

Figure 6. Membership function of the fuzzy logic control MPPT approach (a) Del_P (b) Del_V (c) Del_V $\mathrm{ref}_{\text {ref }}$

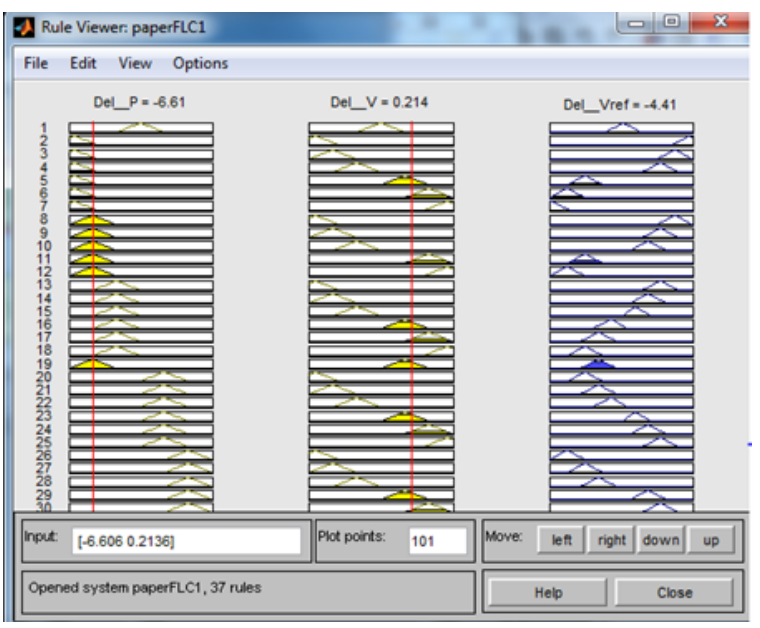

(a)

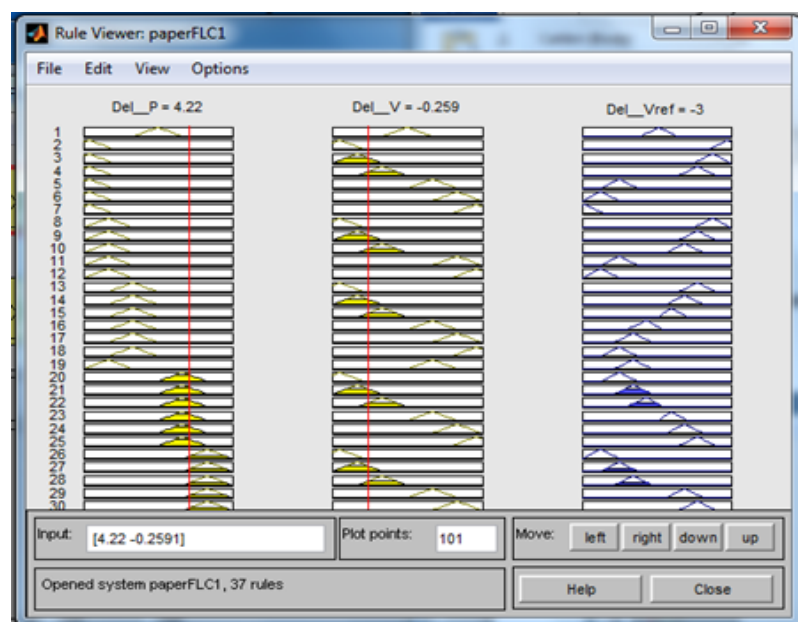

(b)

Figure 7. Rule viewer of the designed new fuzzy logic controller: (a) I/P1 (Del_P) is negative side, (b) IP2 (Del_V) is negative side

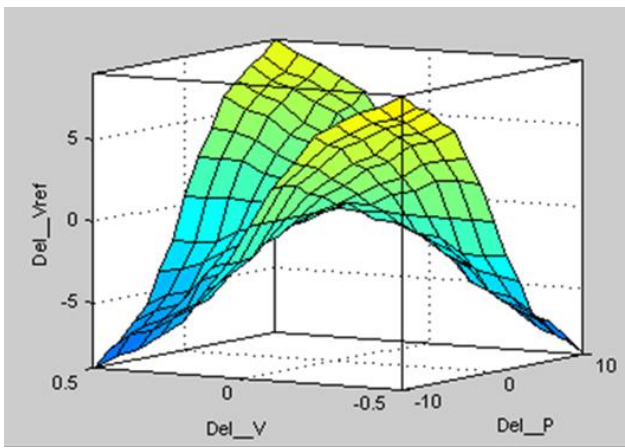

Figure 8. Surface view of designed new fuzzy logic controller 


\section{SIMULATION RESULTS}

The simulation introduced in Figure 9 belonged to output of PV panel and converter of power, current and voltage under constant temperature and irradiation conditions. The figures are clear that the limitation of traditional perturb and observe technique appeared where the reference losses the best point at abrupt irradiation varying. Moreover, at linearly irradiation changing, shows in Figure 10 is clear that the existing perturb and observe method lost the best point and caused fluctuations in the steady state while these drawbacks have been resolved for the suggested new fuzzy logic control based MPPT approach [22]. In both previous figures, the designed fuzzy based maximum power point tracking controller exhibited faster response in the stable steady-state and transient response [21-23]. Furthermore, the fluctuations disappeared, associated with the traditional perturb and observe approach. The fuzzy membership functions (MF) for two inputs and single output are written as given in appendix.

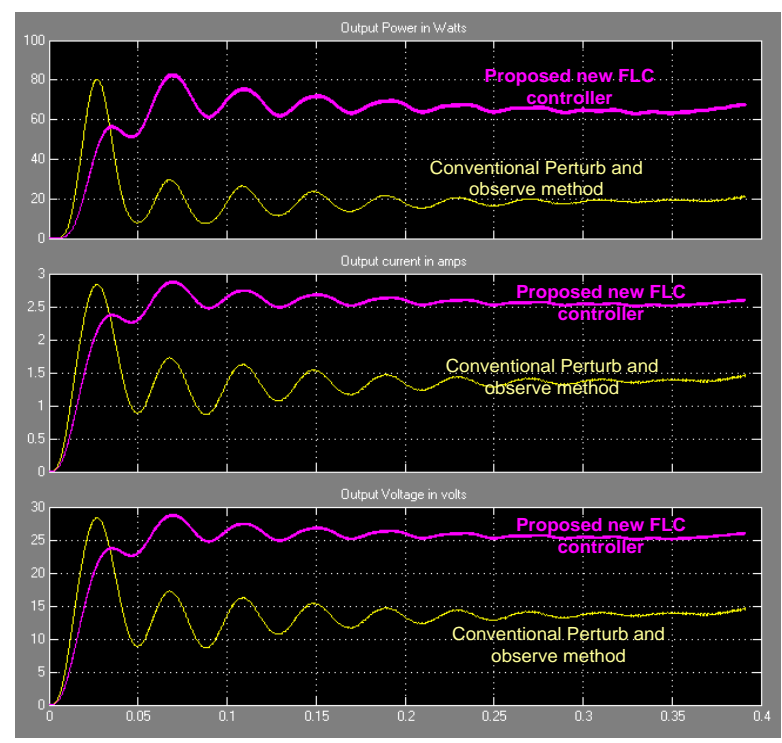

Figure 9. Converter output waveform of power, current and voltage under constant irradiation conditions

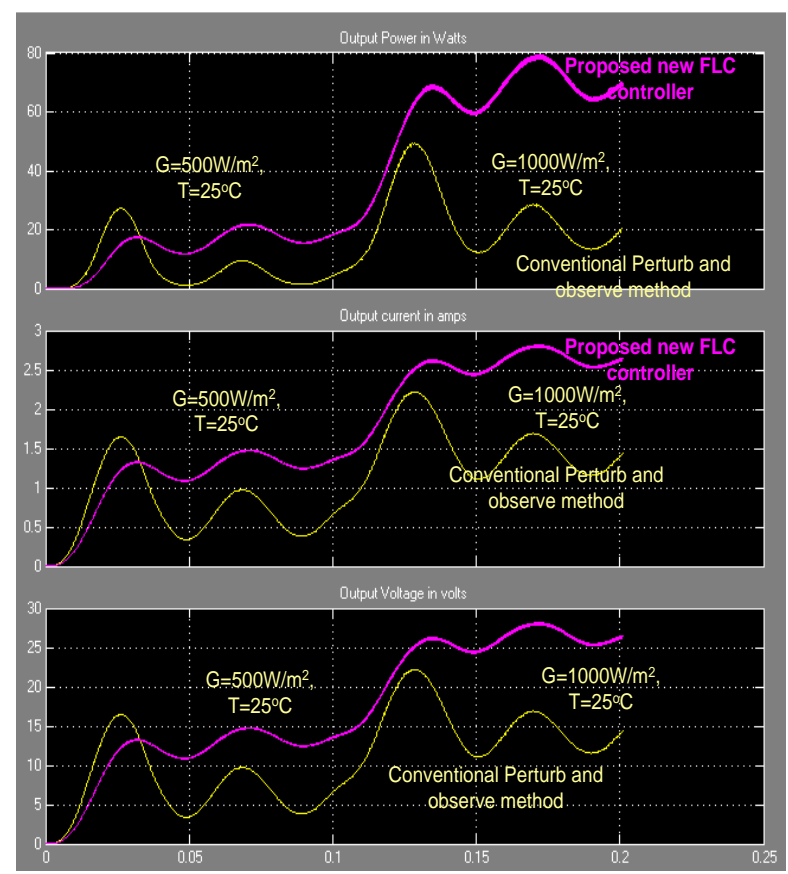

Figure 10. Converter output waveform of power, current and voltage under sudden varying irradiation conditions 


\section{CONCLUSION}

In this paper has introduced an improved Perturb and Observe ( $\mathrm{P} \& \mathrm{O})$ search method has been modified based on fuzzy logic control for maximum power point tracking under rapidly varying weather circumstances. The designed maximum power extracting (MPE) method was implemented by fuzzifying the rules of improved $\mathrm{P} \& \mathrm{O}$ search approach to reduce its weaknesses, with a relatively simple method. A fast converging and accurate to MPE has been presented by Fuzzy Logic Control (FLC) tracker during both steady-state and changing weather circumstances compared to conventional MPPT approaches. Simulink practical experiments model were used to verify the output of conventional $\mathrm{P} \& \mathrm{O}$ and the suggested approach. The outcomes of the designed MPPT exhibit less fluctuation around the maximum peak point under steadystate conditions, a faster converging speed, and no deviation from the peak point during changing weather circumstances. The effectiveness and feasibility of the designed FLC approach were evaluated with dissimilar simulation studies and compared with the existed MPPT techniques.

\section{REFERENCES}

[1] Ahmad H. El Khateb, et al, "Fuzzy logic control approach of a maximum power point employing SEPIC converter for standalone photovoltaic system," Proceeding of the 3rd International Conference on Sustainable Future for Human Security, pp.529 - 536, doi: 10.1016/j.proenv.2013.02.068, 2013.

[2] Ali Akbar Ghassami, et al., "A high performance maximum power point tracker for PV systems," Electrical Power and Energy Systems, vol.53, pp.237-243, 2013.

[3] Arulmurugan, R, "Comparative evaluation of new FLC controller based MPPT for a DC to DC buck-boost zeta converter," WSEAS Transactions on power systems, vol.11, pp.27-34, E-ISSN: 2224-350X, 2016.

[4] M. H. Rashid, "Power Electronics, Circuits Devices and Applications," Academic Press, 2006.

[5] R.Arulmurugan and Dr.N.Suthanthiravanitha, "Model and design of fuzzy-based Hopfield NN tracking controller for standalone PV applications," Elsevier -International Journal of Electric Power Systems Research (IJEPES), vol.120, pp.184-193, DOI 10.1016/j.epsr.2014.05.007Impact, 2015.

[6] N. Mutoh, M. Ohno, and T. Inoue, "A method for MPPT control while searching for parameters corresponding to weather conditions for PV generation systems," IEEE transactions industrial electronics, vol.53, no.4, pp.1055-1065, Aug. 2006.

[7] R. Arulmurugan, et al., "Tracking of photovoltaic power system with new Fuzzy Logic Control strategy," Journal of Electrical Engineering (JEE), Volume 14, 2014-Edition: 4, pp.1-10. ISSN 1582-4594, 2014.

[8] Arulmurugan, R\&Venkatesan, T, "Research and Experimental Implementation of a CV-FOINC Algorithm Using MPPT for PV Power System,” Journal of Electrical Engineering and Technology, vol.10, no.1, pp. 30-40, 2015.

[9] B. N. Alajmi, et al., "Fuzzy-Logic-Control Approach of a Modified Hill-Climbing Method for Maxmum Power Point in microgrid standalone photovoltaic system," IEEE Trans. Power Electronics, vol.26, no.4, pp.1022-1030, 2011

[10] R.Arulmurugan, et al, "Optimal Design of DC to DC Boost Converter with Closed Loop Control PID Mechanism for High Voltage Photovoltaic Application," International Journal of Power Electronics and Drive System (IJPEDS), Vol.3, No.4, pp. 434-444, ISSN: 2088-8694,with scopes index. DOI:10.11591/ijpeds.v2i4.2126, December 2012.

[11] R.Arulmurugan, et al., "An Intelligent novel FLC based FOINC MPPT technique for PV applications", International Conference on Advances in Electrical Engineering at VIT University, Vellore, India on January 9-11, pp 1-6. Index in IEEE Xplore, DOI:10.1109/ICAEE.2014.6838541, 2014.

[12] R.Arulmurugan, et al., "Intelligent fuzzy MPPT controller using analysis of DC-DC buck converter for PV power system applications," IEEE International conference on PRIME 2013 at Periyar University, Salem, on Feb 22 - 23, Index in IEEE Xplore, DOI:10.1109/ICPRIME.2013.6496477, 2013.

[13] C. Emmott, B. Azzopardi, N. Espinosa, R. Garcia-Valverde, A. Urbina, J. Mutale, F. C. Krebs, and J. Nelson, "Economical assessment of solar electricity from organic photovoltaic systems," in Renewable Power Generation (RPG 2011), IET Conference on. IET, 2011, pp. 1-2, 2011.

[14] Z. Liang, R. Guo, J. Li, and A. Q. Huang, "A high-efficiency pv module integrated dc/dc converter for pv energy harvest in freedom systems," Power Electronics, IEEE Transactions on, vol. 26, no. 3, pp. 897-909, 2011.

[15] T. Esram and P. L. Chapman, "Comparison of photovoltaic array maximum power point tracking techniques," Energy conversion, IEEE transactions on, vol. 22, no. 2, pp. 439-449, 2007.

[16] Chia-Hung Lin, Cong-Hui Huang, Yi-Chun Du, Jian-Liung Chen, "Maximum photovoltaic powe tracking for the PV array using the fractional-order incremental conductance method," Applied Energy, 88, pp.4840-4847, 2011.

[17] Dwi Ana Ratna Wati, Wahyudi Budi Pramono, Raditya Dwi Gangsar Wibowo, "Available online at Design and implementation of fuzzy logic controller based on incremental conductance algorithms for photovoltaic power optimization," Proceeding of International Conference on Sustainable Energy Engineering and Application Inna Garuda Hotel, Yogyakarta, Indonesia, pp.45-49, ISBN 978-602-18167-0-7, 6-8th Nov 2012.

[18] Azadeh Safari and Saad Mekhilef, "Simulation and Hardware Implementation of Incremental Conductance MPPT With Direct Control Method Using Cuk Converter," IEEE Transactions on Industrial Electronics, vol. 58, no. 4,pp 1154-1162, April 2011. 
[19] Trishan Esram, Student Member, IEEE, and Patrick L. Chapman, "Comparison of Photovoltaic Array Maximum Power Point Tracking Techniques," IEEE Transactions on Energy Conversion, vol. 22, no. 2, pp.439-450, June 2007.

[20] Ahmad Al Nabulsi and Rached Dhaouadi, "Efficiency Optimization of a DSP-Based Standalone PV System Using Fuzzy Logic and Dual-MPPT Control," IEEE Transactions on Industrial Informatics, vol. 8, NO. 3, pp. 573-585, August 2012.

[21] Mustapha Elyaqouti, et al, "Implementation in Arduino of MPPT Using Variable Step Size P\&O Algorithm in PV Installations," International Journal of Power Electronics and Drive System, Vol. 8, No. 1, pp. 434 443, March 2017.

[22] Chandani Sharma, Anamika Jain, "Performance Comparison of PID and Fuzzy Controllers in Distributed MPPT," International Journal of Power Electronics and Drive System, Vol. 6, No. 3, pp. 625 635, September 2015

[23] Omar Mohammed Benaissa, et al, "Modeling and Simulation of Grid Connected PV Generation System Using Matlab/Simulink," International Journal of Power Electronics and Drive System, vol.8, No.1, 392-401, 2017.

\section{APPENDIX} appendix.

The fuzzy membership functions (MF) for two inputs and single output are written as given in

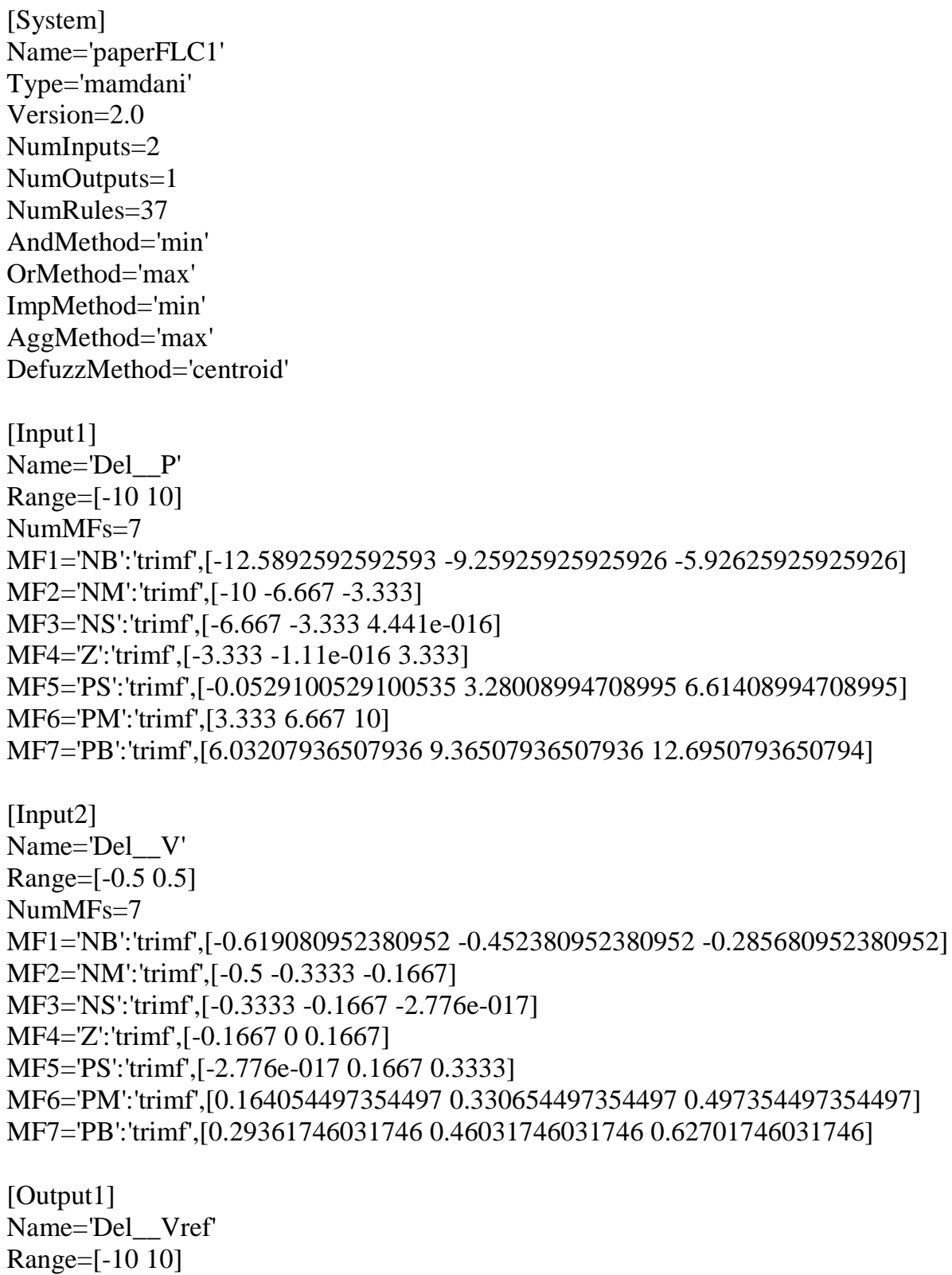


NumMFs $=11$

MF1='NBB':'trimf',[-12.1296296296296 -9.62962962962963 -7.12962962962963]

MF2='NB':'trimf', $[-10-7.5-5]$

MF3='NM':'trimf', [-7.5 -5 -2.5]

MF4='NS':'trimf',[-5.63492063492063 -3.13492063492063 -0.634920634920634]

MF5='Z':'trimf',[-2.18253968253968 0.317460317460316 2.81746031746032]

MF6='PS':'trimf',[1.26984126984127 3.76984126984127 6.26984126984127]

MF7='PM':'trimf',[2.97619047619048 5.47619047619048 7.97619047619048]

MF8='PB':'trimf', [5 7.510$]$

MF9='PBB':'trimf',[7.07671957671958 9.57671957671958 12.0767195767196]

MF10='PSL':'trimf',[0.291040211640209 1.95164021164021 4.15343915343915]

MF11='NSL':'trimf',[-3.99021164021164 -1.38321164021164 0.929788359788357] 